\title{
Feature extraction algorithms from MRI to evaluate quality parameters on meat products by using data mining
}

\author{
Daniel Caballero \\ Department of Computer Science, Research Institute of meat and meat product (IProCar), Cáceres, Spain \\ Advisor and Co-Advisor: Andrés Caro, Teresa Antequera and Trinidad Pérez-Palacios \\ Date and location of PhD thesis defence: 29 May 2017, University of Extremadura.
}

Permanent link of thesis: dehesa.unex.es/handle/10662/5796

Received 27 June 2017; accepted 8 October 2017

\begin{abstract}
This thesis proposes a new methodology to determine quality parameters of meat products (Iberian loin and ham) in a non-destructive way.

Firstly, Magnetic Resonance Imaging (MRI) are obtained from meat products, evaluating three acquisition sequence (Spin Echo -SE-, Gradient Echo-GE- and Turbo 3D -T3D-). Later, MRI is analysed by applying different texture algorithms (GLCM, GLRLM and NGLDM) and fractals algorithms (CFA, FTA and OPFTA); the last two ones have been developed in this thesis. These algorithms extract texture features from the MRI. At the same time, the meat products are also analysed by means of physico-chemical and sensory techniques. Finally, different data mining techniques are applied on all obtained data: deductive (Multiple linear regression MLR-), classification (Decision trees -DT- and Rules-based systems -RBS-) and prediction techniques (MLR and Isotonic regression $-\mathrm{IR}-$ ).

The accuracy of the analysis of quality parameters is affected by the MRI acquisition sequence, the algorithm used to analyse them and the data mining technique applied. In general, the use of SE as MRI acquisition sequence, and GLCM or OPFTA as image analysis algorithm could be indicated. Considering the data mining techniques, MLR and DT are appropriate, respectively, to deduce physico-chemical parameters and to classify as a function of salt content. Regarding to the predictive technique, MLR could be indicated. It offers reliable equations to determine the quality parameters, and, allows analysing the quality of meat products in a nondestructive, efficient, effective and accurate way.
\end{abstract}

Key Words: MRI, Computer Vision Algorithms, Data Mining.

\section{Introduction}

Iberian dry-cured meat products are highly appreciated by consumers all over the world. Quality attributes of Iberian dry-cured meat products depend on the characteristics of the raw material and the processing conditions.

Traditional methods for evaluating the quality characteristics of Iberian dry-cured meat products throughout the whole processing are tedious and time-consuming, and involve the destruction of the pieces

Correspondence to: dcaballero@unex.es

Recommended for acceptance by Anjan Dutta and Carles Sánchez

https://doi.org/10.5565/rev/elcvia. 1100

ELCVIA ISSN: 1577-5097

Published by Computer Vision Center / Universitat Autònoma de Barcelona, Barcelona, Spain 
for carrying out the analysis. The use of non-destructive techniques, such as Charge-Coupled Device Cameras (CCD), Computed Tomography (CT) and MRI have been suggested as an alternative to conventional methods.

MRI is a non-destructive, non-invasive, non-ionizing and innocuous technique. Different acquisition sequences of MRI, such as SE, GE and T3D [1] and they can be weighted with different spin lattice relaxation time, T1 and T2 [2]. The most widely applied combination in the scientific literature is SE as acquisition sequence and $\mathrm{T} 1$ as a relaxation time.

For analysing the obtained images, all these studies have applied classical texture algorithm [3-4]. Most of these studies on MRI used common statistical tools, such as Pearson's correlations coefficients or principal components analysis [3-4].

Recently, some data mining techniques have been tested to predict some quality attributes of meat products [5-6]. Data mining is one of the steps of a larger process known as knowledge discovery in databases (KDD) [7].

From the described approach, this thesis seeks to advance in three ways: image acquisition, image analysis by using computer vision techniques and data treatments, being the main objective:

The proposal of a new methodology based on the use of MRI, the development of new algorithms for the image analysis, and the application of data mining techniques in order to determine quality characteristics of meat products in a non-destructive way.

\section{Motivation}

This thesis is developed in a multidisciplinary scope, continuing the research field "Characterizing meat products throughout Magnetic Resonance Imaging (MRI) analysis" between researchers of the Media Engineering Group (GIM) from School of Technology and researchers of the Technology and Quality of Food (TECAL) from the Faculty of Veterinary Medicine, both from the University of Extremadura.

The link between this two groups focuses on the use of MRI for the study of the quality characteristics of meat products of non-destructive way, approaching this research from two areas of complementary knowledge.

This line of interdisciplinary research is defined by the progress in three ways: acquisition of MRI, image analysis by means of computer vision techniques and data processing.

\section{Contribution}

The main contribution of this thesis is the development of two new computer vision algorithms: FTA [8] and OPFTA [9]. They allow analysing MRI images, properly, noting OPFTA for its simplicity and lower computational cost.

The new methodology developed in this doctoral thesis was initially applied for researching purposes. But, currently, this methodology of analysis is offered to the industry by an external service of the University of Extremadura (Animal Source Foodstuffs Innovation Services -SiPA-).

\section{Brief method}

The general procedure consists of obtaining MRI of meat products, and applying different computer vision algorithms (texture and fractal approaches, mainly), which allow the extraction of sets of computational features. Figure 1 shows the design of the proposed procedure.

To achieve this, different research has been done, based on:

- MRI Scanners

○ High Field (Philips Gyroscan NT Intera 1.5 T)

○ Low Field (Esaote VET-MR E-SCAN XQ 0.18 T)

- MRI acquisition sequences 
$\begin{array}{ll}\circ & \text { GE } \\ \circ & \text { SE } \\ \circ & \text { T3D }\end{array}$

- Texture algorithms

- Grey Level Co-ocurrence Matrix (GLCM)

- Grey Level Run Length Matrix (GLRLM)

○ Neighbouring Grey Level Dependence Matrix (NGLDM)

- Fractal algorithms

- Classical Fractal Algorithm (CFA)

- Fractal Texture Algorithm (FTA)

$\circ$ One Point Fractal Texture Algorithm (OPFTA)

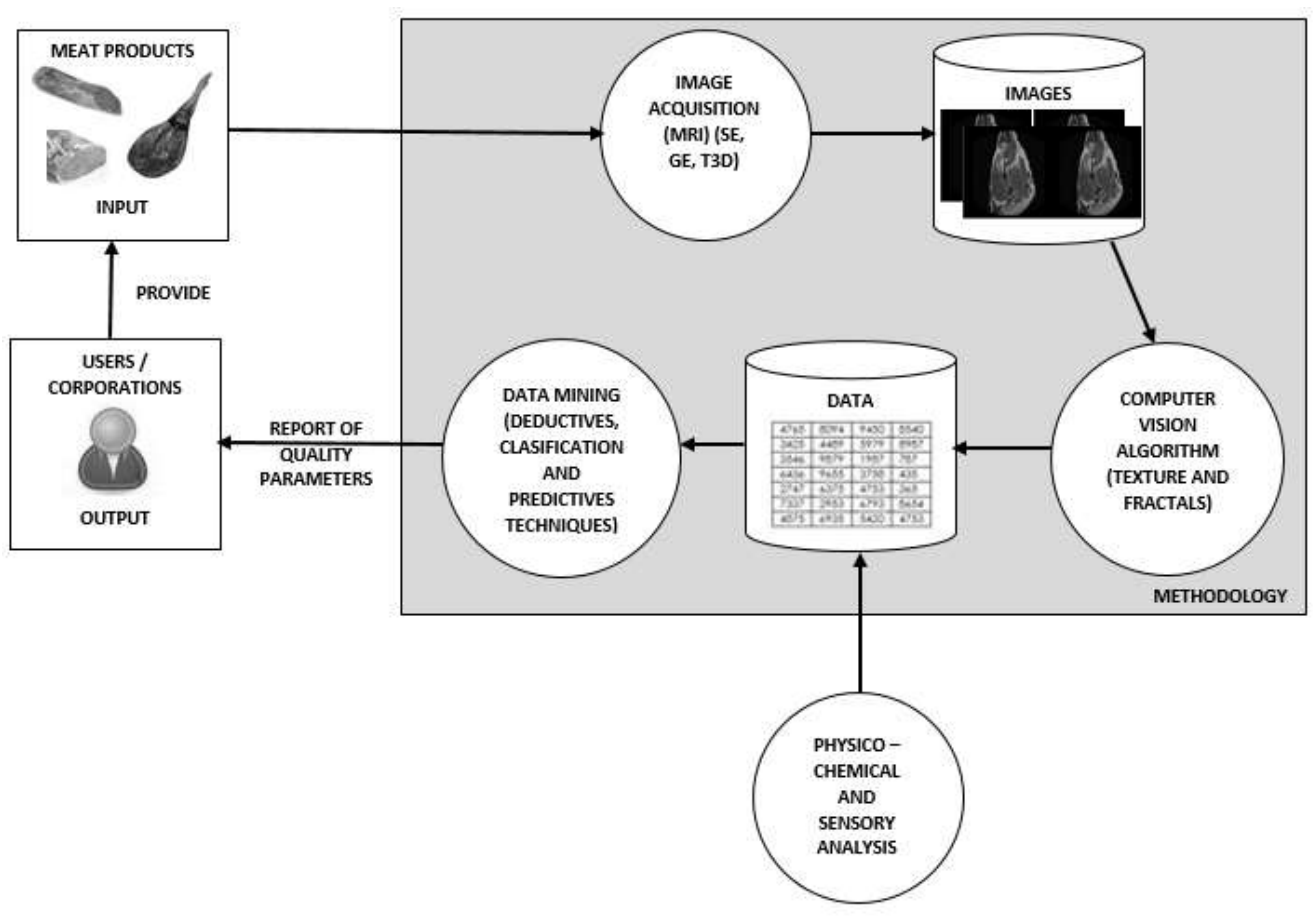

Figure 1.- Design of the proposed methodology.

At the same time that the MRI are analysed, the Iberian loin and ham were also analysed by means of physico-chemical and sensory techniques. Databases were constructed with all these data. Different data mining techniques were applied on them: deductive (MLR) [10], classification (DT and RBS) [11] and prediction techniques (IR and MLR) [12-15].

\section{$5 \quad$ Brief results}

Two new algorithms have been developed in this thesis, FTA [8] and OPFTA [9]. Both of them show better coefficient results than texture and CFA algorithms, noting OPFTA for its simplicity and lower computational cost.

The accuracy of the analysis of the quality parameters of Iberian ham and loin is affected by the MRI acquisition sequence, the algorithm used to analyse them and the data mining technique applied. Considering the data mining techniques, MLR and DT are appropriate, respectively, to deduce physico-chemical parameters of hams [10], and to classify as a function of salt content in hams [11]. Regarding to the predictive technique, MLR allows obtaining equations to determine the physico-chemical characteristics and sensory attributes of Iberian hams [12] and loins [13-15] with a high degree of reliability, and analysing the quality of these meat products in a non-destructive, efficient, effective and accurate way. 


\section{Conclusions}

The main conclusion from this thesis has been the proposal of a methodology to determine quality characteristics of meat products (Iberian loin and ham) in a non-destructive way. This has been achieved by using MRI of meat products, processing the MRI by applying different algorithms and analysing the obtained data by means of data mining techniques. In particular, the application consists of using of SE as MRI acquisition sequence, GLCM or OPFTA as image analysis algorithm and MLR as data mining technique, in order to obtain prediction equations of physico-chemical characteristics and sensory attributes of Iberian loins and hams with a high degree of reliability. In this way, the quality of these products is determined in a non-destructive, fast and accurate way.

\section{References}

[1] Pérez-Palacios, T., Caballero, D., Caro, A. and Antequera, T. "Low field magnetic resonance imaging and computational texture features to predict moisture and lipid content of loins". IV Farm Animal Imaging Conference. Edinburgh, United Kingdom, 2015.

[2] Fantazzini, P., Bortolotti, V., Garavaglia, C., Gombia, M., Riccardi, S., Schembri, P., Virgili, R. and Bordini, C.S. "Magnetic resonance imaging and relaxation analysis to predict non-invasively and non-destructively saltto-moisture ratios in dry-cured meat", Magnetic Resonance Imaging, 23, 359-361, 2005.

[3] Antequera, T., Caro, A., Rodríguez, P.G., and Pérez-Palacios, T. "Monitoring the ripening process of Iberian ham by computer vision on magnetic resonance imaging", Meat Science, 76, 561-567, 2007.

[4] Manzoco, L., Anese, M., Marzona, S., Innocente, N., Lazaglio, C. and Nicoli, M.C. "Monitoring dry-curing of San Daniele ham by magnetic resonance imaging”, Food Chemistry, 141, 2246-2252, 2013.

[5] Alamprese, C., Amigo, J.M., Engelsen, J.M., and Casiraghi, E. "Identification and quantification of turkey meat adulteration in fresh, frozen-thawed and cooked minced beef by FT-NIR spectroscopy and chemometrics", Meat Science, 121, 175-181, 2016.

[6] Cortez, P., Portelinha, S., Rodrigues, S., Cadavez, V. and Teixeira, A. "Lamb meat quality assessment by support vector machines", Neural Processing Letters, 24, 41-51, 2006.

[7] Fayyad, U., Piatetsky-Shapiro, G., and Smyth, P. "From data mining to knowledge discovery in databases", American Association for Artificial Intelligence, 17, 37-54, 1996.

[8] D. Caballero, A. Caro, M. M. Ávila, P. G. Rodríguez, T. Antequera, and T. Pérez-Palacios "New fractal features and data mining to determine food quality based on MRI", IEEE Latin American Transactions, 15(9), 17771784, 2017.

[9] D. Caballero, A. Caro, J.M. Amigo, A.B. Dahl, B.K. Ersboll and T. Pérez-Palacios "Development of a new fractal algorithm to predict quality traits of MRI loins", Lecture Notes in Computer Science, 10424, 208-218, 2017.

[10] T. Pérez-Palacios, D. Caballero, A. Caro, P.G. Rodríguez and T. Antequera "Applying data mining and Computer Vision Techniques to MRI to estimate quality traits in Iberian hams", Journal of Food Engineering, 131, 82-88, 2014.

[11] D. Caballero, A. Caro, P.G. Rodríguez, M.L. Durán, M.M. Ávila, R. Palacios, T. Antequera and T. PérezPalacios "Modeling salt diffusion in Iberian ham by applying MRI and data mining", Journal of Food Engineering, 189, 115-122, 2016.

[12] D. Caballero, T. Antequera, A. Caro, M.L. Durán and T. Pérez-Palacios. "Data mining on MRI-Computational Texture Features to predict sensory characteristics in ham", Food and Bioprocess Technology, 9, 699-708, 2016.

[13] T. Pérez-Palacios, D. Caballero, T. Antequera, M.L. Durán, M.M. Ávila and A. Caro "Optimization of MRI acquisition and texture analysis to predict physico-chemical parameters of loins by data mining", Food and Bioprocess Technology, 10, 750-758, 2017.

[14] D. Caballero, T. Pérez-Palacios, A. Caro, J.M. Amigo, A.B. Dahl, B.K. Ersboll and T. Antequera, "Prediction of pork quality parameters by applying fractals and data mining on MRI", Food Research International, 99, 739$747,2017$.

[15] D. Caballero, T. Antequera, A. Caro, M.M. Ávila, P.G. Rodríguez and T. Pérez-Palacios. "Non-destructive analysis of sensory traits of dry-cured loins by MRI-computer vision techniques and data mining". Journal of the Science of Food and Agriculture, 97, 2942-2952, 2017. 